\title{
Dynamic crack-interface interactions in SGP laminated glass: an experimental investigation
}

\author{
Chengliang $\mathrm{Xu}^{1}$, Ye Yuan ${ }^{1,2 *}$, Chunlei Zhao ${ }^{1}$, P.J. Tan ${ }^{2}$, Xiaoqing $\mathrm{Xu}^{1}$, Yibing $\mathrm{Li}^{1}$ \\ ${ }^{1}$ State Key Laboratory of Automotive Safety \& Energy, Department of Automotive Engineering, \\ Tsinghua University, Beijing 100084, China \\ ${ }^{2}$ Department of Mechanical Engineering, University College London, \\ Torrington Place, London WC1E 7JE, UK
}

\section{Abstract}

The damage mode (crack arrest or bifurcation) that develops in SentryGlas ${ }^{\circledR}$ Plus (SGP) laminated glass under dynamic impact loading is investigated experimentally in this paper. An optical caustic method is employed - which allows simultaneous measurements of the spatial and temporal evolution of crack paths and the dynamic stress intensity factor at the crack tip(s) - to elucidate the effects of interface location and impact kinetic energy upon the fracture morphology, crack propagation velocity and the dynamic stress intensity factor in SENB (single-edge notch bend) test specimens loaded in three-point bending by a dropweight impact system. Results reveal that there is a critical distance, between the interface and pre-crack tip, below which the propagating mode-I crack is arrested by the interface; otherwise, bifurcated mixed-mode cracks will always appear in the impacted glass layer. A maximum dynamic stress intensity factor criterion is shown to be capable of predicting whether bifurcated mixed-mode cracks would appear in the aforesaid.

9 Keywords: Dynamic fracture, Crack-interface interaction, Optical caustic method,

10 Laminated glass, Dynamic stress intensity factor

\section{1. Introduction}

12 The superior damage-tolerance of laminated glass compared to its monolithic counterpart is

13 the reason for their widespread use as safety glasses in the automotive (Liu et al., 2016; Yuan

14 et al., 2017b) and civil (Zhang et al., 2013; Yuan et al., 2017a) industries. Laminated glass

15 typically consists of two brittle glass layers bonded by a polymeric interface (or interlayer)

\footnotetext{
${ }^{*}$ Corresponding author

Email addresses: yuanye16@tsinghua.edu.cn (Ye Yuan $\left.{ }^{1,2}\right)$, pj.tan@ucl.ac.uk (P.J. $\left.\operatorname{Tan}^{2}\right)$
} 
that has excellent optical and adhesive properties, such as SentryGlas ${ }^{\circledR}$ Plus (SGP). Even after cracks have permeated both brittle glass layers (or plies) in the laminated glass, it is still able to offer protection to the target space ahead from penetration by foreign objects due to the ductile interlayer, which also serves to bind the majority of the glass splinters prior to its rupture. There are numerous recent studies examining the in-plane cracking of individual glass plies in laminated glass; for example, by Chen et al. (2013, 2014); Xu et al. (2017, 2016). Instead, this study seeks to further elucidate the conditions (specifically on interface location and impact kinetic energy of a projectile) that affects crack growth across the interface in SGP laminated glass through an optical caustic method, which allows the concurrent measurements of dynamic stress intensity factor and crack path.

\begin{tabular}{|ll|}
\hline Nomenclature \\
$C_{d}$ & dilatational wave speed \\
$C_{s}$ & shear wave speed \\
$C_{t}$ & optical stress constant of glass \\
$D(t)$ & transverse diameter of caustic \\
$E$ & Young's modulus of glass \\
$E_{0}$ & impact kinetic energy \\
$F(V)$ & correction factor for crack velocity \\
$h_{t}$ & total height of specimen \\
$h_{d}$ & drop-height \\
$K_{I}^{d}(t)$ & dynamic stress intensity factor \\
$l$ & crack length \\
$\mathrm{MDSIF}$ & maximum dynamic stress intensity factor \\
$R(V)$ & Rayleigh function \\
$t$ & time \\
$V(t)$ & crack velocity \\
$x, y$ & in-plane coordinates \\
$z_{0}$ & distance between the specimen front surface and the reference plane \\
$\delta$ & correction factor to offset caustic distortion \\
$\Delta h$ & distance from pre-cracked tip to interface \\
$\nu$ & Poisson's ratio of glass \\
$\Pi$ & energy dissipation \\
\hline
\end{tabular}

27 A considerable body of literature already exists - see Sundaram and Tippur (2016b,a, 2017); 28 Yan et al. (2018); Needleman (2018); Lee et al. (2007); Park and Chen (2011) and Xu and 29 Rosakis (2003) to name a few - on dynamic crack-interface interactions in laminated (or so layered) structures. Different damage modes can develop in a laminated glass. If the initial 31 transverse crack, that traverses through the brittle support layer, is arrested at the adhesive 
interface (see schematic in Fig. 1), this is known as a crack inhibition mode (Theocaris and Milios, 1981; Dally and Kobayashi, 1978; Lee et al., 2007). In the subsequent crack-interface interaction, provided there is sufficient energy to drive further crack growth, different damage modes may develop as follows: delamination - crack deflection into the interface (He and Hutchinson, 1989; Clegg et al., 1990; Sundaram and Tippur, 2016b); penetration - the initial crack jumps across the interface and reappears at the impacted layer (Siegmund et al., 1997; $\mathrm{Xu}$ and Rosakis, 2003); branching - bifurcation of the initial crack into multiple mixed-mode cracks that emanate from where the initial crack first interacts with the adhesive interface (Shaw et al., 1993; Theocaris and Demakos, 1986; Park and Chen, 2011; Sundaram and Tippur, 2016b).

Various experimental techniques were previously employed to study crack-interface interactions. Dally and Kobayashi (1978) studied crack inhibition in duplex specimens using photoelastic method. They reported that the instantaneous stress intensity factor of the propagating crack needs to be sufficient high to cause crack branching; otherwise, the crack is arrested by the interface. High-speed photography was employed by Park and Chen (2011) to visualise the dynamic crack-interface interactions, driven by projectile impact, in a laminated glass. They found that crack penetration and branching are controlled by interfacial conditions that depends on the interface thickness, adhesive strength and surface finish. Increasing interface thickness increases the crack-interface interaction time, which leads to the appearance of more branched cracks at increasingly greater crack-branching angle. However, the higher stresses induced by dynamic loading has the opposite effect of reducing this branching angle. More recently, Sundaram and Tippur (2016b) investigated the effects of interface location on crack penetration and bifurcation behaviours in PMMA bilayers using optical measurements based on Digital Gradient Sensing (DGS). They reported that the crack path selection at the interface and, subsequently, the second layer are strongly affected by the location of the interface.

In this paper, we investigated the interaction of a dynamic crack with a perpendicular interface in SGP laminated glass by using an optical caustic method, first introduced by Theocaris (1970). The caustic method - it has previously been employed to investigate dynamic fracture in composites and polymer (Theocaris and Demakos, 1986; Theocaris and Milios, 1981; Tang, 2014; Yao et al., 2004; Yao and Xu, 2011; Hao et al., 2016b, 2015) - is used here, for the first time, to visualise the crack path(s) and to measure the dynamic stress intensity factor at the crack tip. The results will be used to elucidate the effects of interface location and impact kinetic energy on the fracture morphology, crack propagation velocity and the dynamic stress intensity factor in SGP laminated glass under a three-point-bending test configuration. 


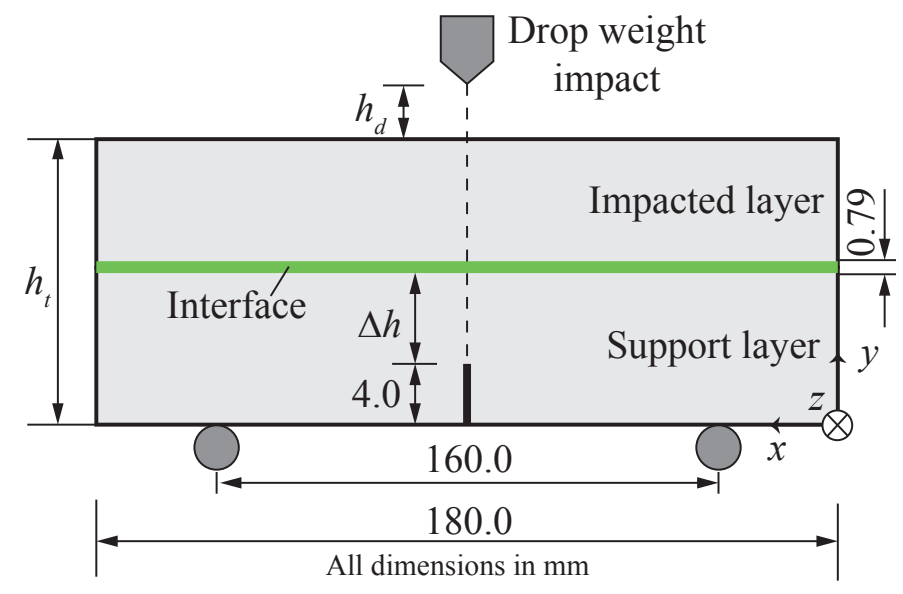

Figure 1: Schematic of the SENB (single-edge notched bend) laminated glass specimen loaded in three-point bending by a drop-weight impact system. Plane of loading and geometric symmetry is denoted by the vertical dashed line.

\section{2. Experiments}

\subsection{Specimens and loading}

Two $180 \mathrm{~mm}$ wide soda-lime glass plates of $5 \mathrm{~mm}$ thickness were affixed to a $180 \mathrm{~mm} \times 5 \mathrm{~mm}$ $\times 0.79 \mathrm{~mm}$ (width $\times$ thickness $\times$ height) adhesive interface in an edge-to-edge configuration to form the laminated glass with a total height $h_{t}$ of between $40.79-60.79 \mathrm{~mm}$, as shown schematically in Fig. 1. The interface was made of SentryGlas ${ }^{\circledR}$ Plus (SGP), a transparent ionoplast material manufactured by DuPont, USA. The material properties of the soda-lime glass and SGP are listed in Table 1. Whilst glass can generally be considered to be linear elastic and strain-rate insensitive (Liu et al., 2016; Xu et al., 2017), by contrast, SGP exhibits non-linear elasto-plastic response and is highly strain-rate dependent (Bennison et al., 2005; Zhang et al., 2015). In general, SGP becomes less ductile with increasing strain rate; for example, its failure strain reduces from about $400 \%$ under quasi-static loading rate to about $150 \%$ at a strain rate of $2000 \mathrm{~s}^{-1}$ (Zhang et al., 2015). Nonetheless, SGP is considerably softer compared to soda-lime glass as seen in Table 1. A single edge-crack (pre-crack) of $4 \mathrm{~mm}$ length and $1 \mathrm{~mm}$ width is introduced at the mid-point (on the bottom face) of the support layer, along the same line of action as the drop weight impactor; this was fabricated using a high pressure water-jet to achieve a smooth finish. The adhesive interface is located at a distance $\Delta h$ from the pre-crack tip. The single-edge notch bend (SENB) specimen used here is a common test geometry for fracture toughness determination. A $0.75 \mathrm{~kg}$ impactor is dropped from different heights $h_{d}$ of between 50 - $200 \mathrm{~mm}$ to give an impact kinetic energy $E_{0}$ that ranges from 0.37 to $1.47 \mathrm{~J}$. 
Table 1: Constituent material properties for the laminated glass

\begin{tabular}{ccccc}
\hline Material & $\begin{array}{c}\text { Density, } \\
\left(\mathrm{kg} / \mathrm{m}^{3}\right)\end{array}$ & $\begin{array}{c}\text { Young's modulus, } \\
(\mathrm{GPa})\end{array}$ & Poisson's ratio & Failure strain \\
\hline Glass (Liu et al., 2016) & 2500 & 70 & 0.227 & $0.1 \%$ \\
SGP (Zhang et al., 2015) & 950 & 0.3 & 0.5 & $\geq 150 \%$ \\
\hline
\end{tabular}

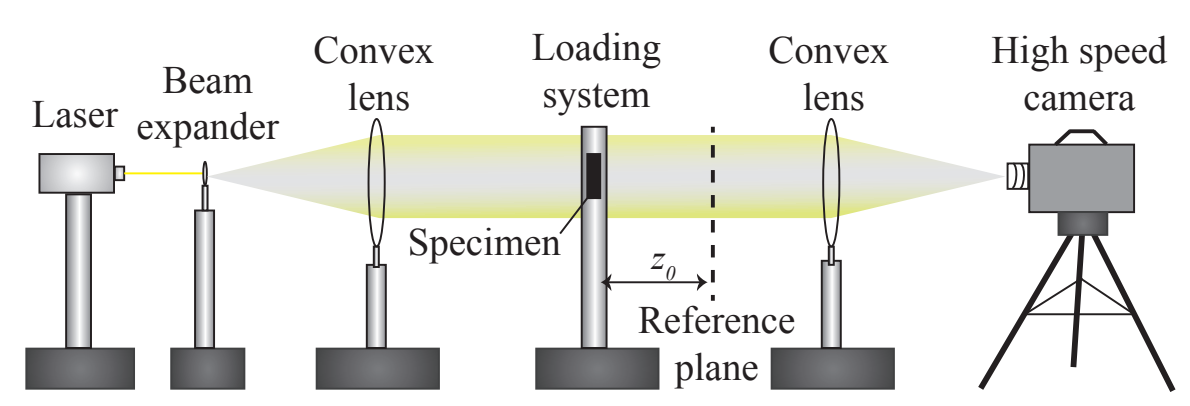

Figure 2: Schematic of experimental set-up for the caustic experiment.

\subsection{Experimental setup}

Figure 2 shows a schematic of the experimental setup used in this study. It comprises of a high-powered laser diode (green laser light is used because it is the colour to which high speed cameras are most sensitive), beam expander, two convex lenses, drop-weight impact system (described in Section 2.1) and a high speed camera.

The beam expander and convex lens were used to generate an expanded collimated light source. When this collimated light penetrates the back face of the test specimen, it becomes deviated by the highly strained zone in the crack-tip vicinity and by the cracked path, which is captured by the high speed camera from which the caustic images of cracked specimens were obtained. A Fastcam-SA5 high speed camera (by Photron Japan, Inc.), with frequency of up to a million frames per second, is used; in our experiments, 4.8 Gigabytes of data per second were recorded, which yields a pixel-to-length ratio of $264.6 \mu \mathrm{m} /$ pixels at a frequency of between 210,000 and 300,000 frames per second, depending on the total height $h_{t}$ of the specimen. The reference plane is located at a distance of $z_{0}=3.1 \mathrm{~m}$ in front of the test specimen.

\subsection{Measurement of the dynamic stress intensity factor}

Following Papadopoulos (1993), the dynamic mode-I stress intensity factor $K_{I}^{d}(t)$ in an optically isotropic material, containing a stationary pre-crack, is given by

$$
K_{I}^{d}(t)=\frac{2 \sqrt{2 \pi} F(V)}{3 z_{0}\left|C_{t}\right| d}\left[\frac{D(t)}{\delta}\right]^{\frac{5}{2}}
$$




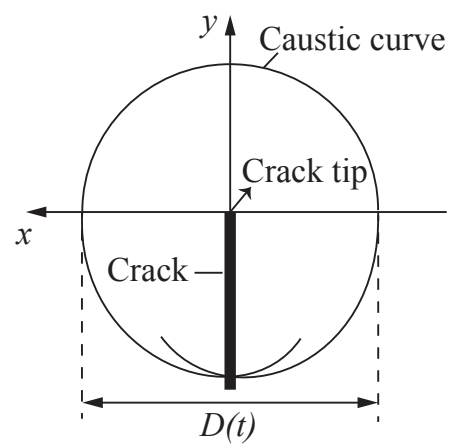

Figure 3: Schematic of mode-I caustic curve at crack tip (Yao et al., 2007).

where $D(t)$ is transverse diameter of the caustic as depicted in Fig. $3 ; C_{t}=-0.225 \times 10^{-11}$ $\mathrm{Pa}^{-1}$ (Gan, 1964) is the optical stress constant for glass; $d=0.005 \mathrm{~m}$ is thickness of the specimen in the transmitted caustic; $z_{0}=3.12 \mathrm{~m}$ is the distance between the reference plane and the specimen plane; $\delta=3.17$ (Yao et al., 2007; Zhang et al., 2007) is a correction factor that offsets the shape distortion in the caustics; $F(V) \approx 1$ is the typical correction factor used for all practical crack velocities $V$ (Yao et al., 2003; Hao et al., 2016a). The dynamic stress intensity factor of the crack tip at time $t$ is obtained by measuring the transverse diameter of the caustics $D(t)$.

\section{Results}

This section presents test data that reveal how different damage regimes (either crack inhibition or branching) may develop depending on two important parameters, viz. interface location and impact kinetic energy, that are pertinent to the assessment of the structural integrity (containment of cracking within the support layer) of laminated glass under impact loading.

\subsection{Effects of interface location $\Delta h$}

The effects of interface location on the crack-interface interaction is first investigated. Figures 4, 5 and 6 show the temporal evolution of caustic spots in three typical specimens with their interface located at $\Delta h=16,26$ and $36 \mathrm{~mm}$, respectively. The experimental photos of caustic spot in specimens with three different interface locations $(\Delta h=16 \mathrm{~mm}, \Delta h=26$ $\mathrm{mm}$ and $\Delta h=36 \mathrm{~mm}$ ) at selected time frames are shown in Figs. 4, 5 and 6 . Note that all specimens shown have identical total height of $h_{t}=60.79 \mathrm{~mm}$ and are subjected to identical impact kinetic energy of $E_{0}=1.47 \mathrm{~J}$, which yields an impact velocity of $1.98 \mathrm{~m} / \mathrm{s}$. The images were recorded using a Fastcam-SA5 high speed camera at 210,000 frames per second.

Time $t=0 \mu$ s corresponds to when cracking first initiates at the pre-crack tip, from where a caustic spot always emanate in all specimens - see Figs. 4a, 5a and 6a. It is evident from 


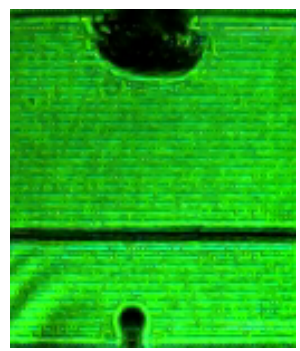

(a) $0 \mu \mathrm{s}$

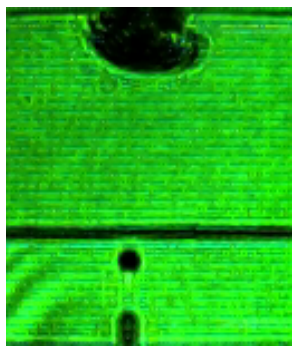

(b) $9.5 \mu \mathrm{s}$

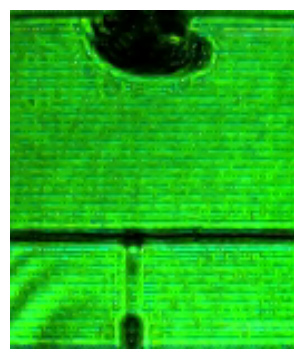

(c) $14.3 \mu \mathrm{s}$

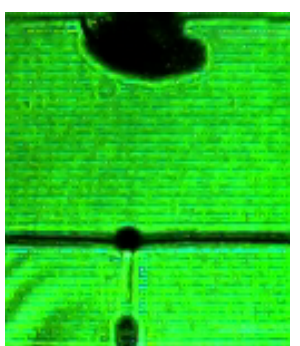

(d) $147.6 \mu \mathrm{s}$

Figure 4: Temporal evolution of caustic spot through the support layer of a specimen $(\Delta h=$ $16 \mathrm{~mm}, h_{t}=60.79 \mathrm{~mm}, h_{d}=200 \mathrm{~mm}$ and $E_{0}=1.47 \mathrm{~J}$ ). The initial mode-I crack in the support layer arrives at the interface at $t=14.3 \mu \mathrm{s}$ (third frame).

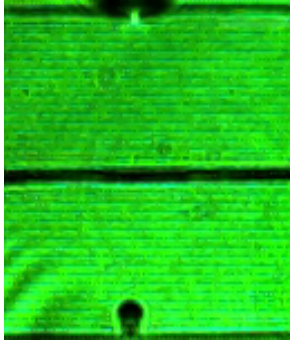

(a) $0 \mu \mathrm{s}$

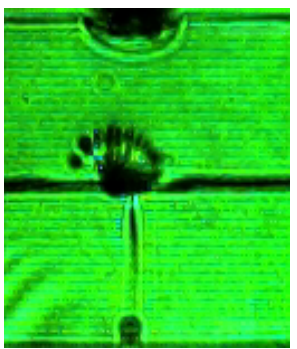

(e) $71.4 \mu \mathrm{s}$

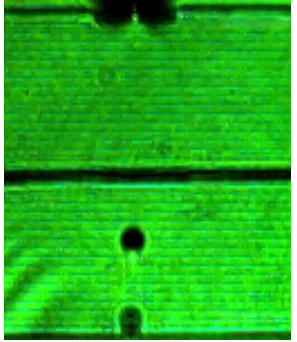

(b) $9.5 \mu \mathrm{s}$

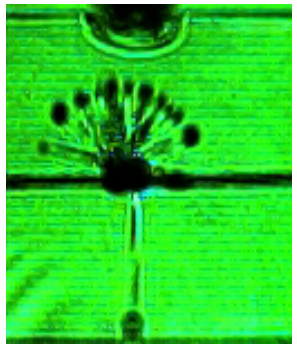

(f) $76.2 \mu \mathrm{s}$

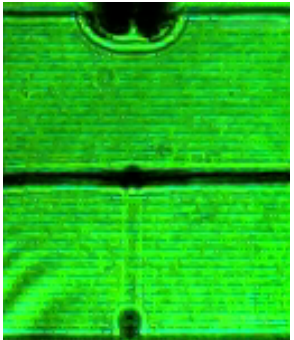

(c) $19.0 \mu \mathrm{s}$

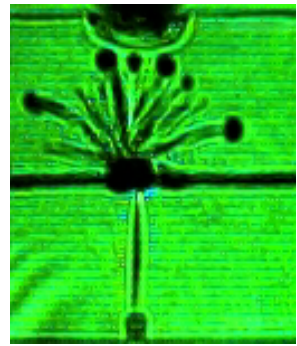

(g) $81.0 \mu \mathrm{s}$

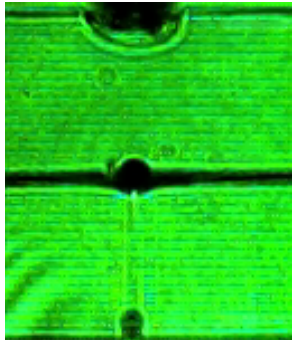

(d) $66.7 \mu \mathrm{s}$

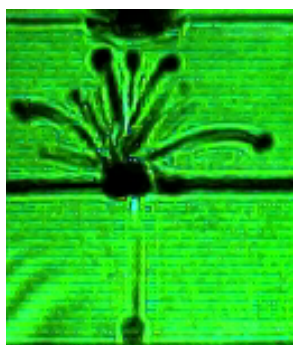

(h) $85.7 \mu \mathrm{s}$

Figure 5: Temporal evolution of caustic spots $\left(\Delta h=26 \mathrm{~mm}, h_{t}=60.79 \mathrm{~mm}\right.$ and $h_{d}=200$ $\left.\mathrm{mm}, E_{0}=1.47 \mathrm{~J}\right)$. Time $t=19.0 \mu \mathrm{s}$ and $t=71.4 \mu \mathrm{s}$ corresponds to the instant when the mode-I crack in the support layer reaches the interface and when multiple mixed-mode cracks first appeared in the impacted layer, respectively.

Figs. 4,5 and 6 that, regardless of interface location $\Delta h$ and the impact kinetic energy $E_{0}$ (to be presented later), cracking in the support layer always occur in mode-I and its ensuing crack path lies nominally along the loading and geometric symmetry line - this does not appear to be affected by transverse shear - until the crack tip starts interacting with the interface. The subsequent crack path(s) that develop differs considerably after this point. For $\Delta h=16 \mathrm{~mm}$, the initial mode-I crack was arrested by the interface and, consequently, 


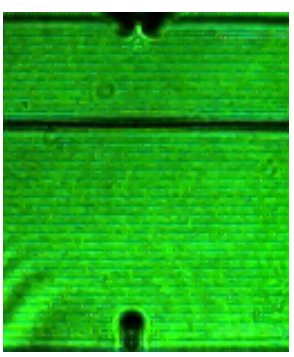

(a) $0 \mu \mathrm{s}$

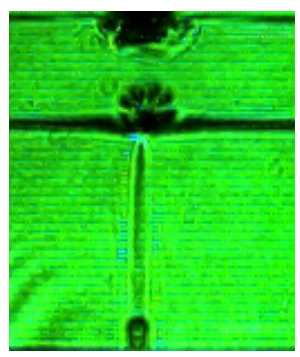

(e) $119.0 \mu \mathrm{s}$

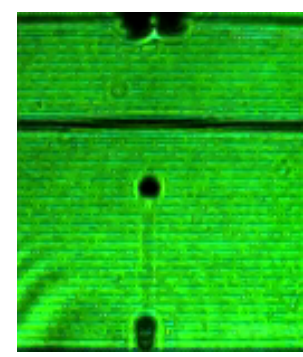

(b) $19.0 \mu \mathrm{s}$

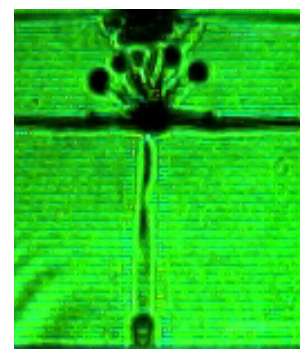

(f) $123.8 \mu \mathrm{s}$

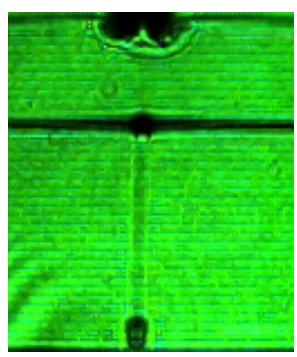

(c) $52.4 \mu \mathrm{s}$

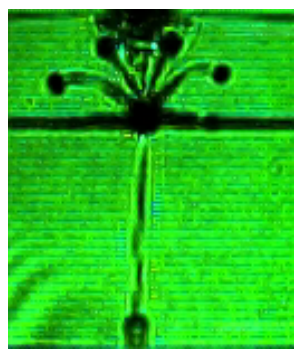

(g) $128.6 \mu \mathrm{s}$

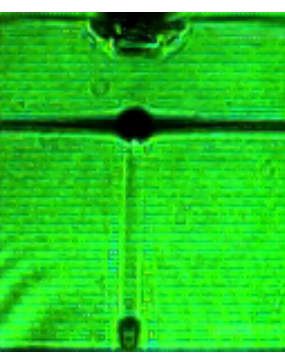

(d) $104.7 \mu \mathrm{s}$

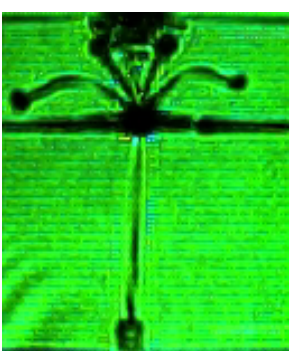

(h) $133.3 \mu \mathrm{s}$

Figure 6: Temporal evolution of caustic spots $\left(\Delta h=36 \mathrm{~mm}, h_{t}=60.79 \mathrm{~mm}\right.$ and $h_{d}=200$ $\left.\mathrm{mm}, E_{0}=1.47 \mathrm{~J}\right)$. Time $t=52.4 \mu \mathrm{s}$ and $t=119.0 \mu \mathrm{s}$ corresponds to the instant when the mode-I crack in the support layer reaches the interface and when multiple mixed-mode cracks first appeared in the impacted layer, respectively.

the impacted layer remains intact (crack-free) - this is known as the crack inhibition mode. By contrast, increasing $\Delta h$ causes cracking to re-initiate in the impacted layer in the form of multiple mixed-mode cracks, where their ensuing crack paths are almost globally symmetric with respect to the plane of symmetry. The mixed-mode cracks, at some stage (see Figs. 5g and $6 \mathrm{~g}$ ), deflect inwards towards the interface, which is a result of transverse shear effect. Crack initiation in the impacted layer occurs at $t=71.4 \mu \mathrm{s}$ and $t=119.0 \mu \mathrm{s}$ in Figs. $5 \mathrm{~d}$ and $6 \mathrm{~d}$, respectively. It is worth noting that in the former, Fig. $5 \mathrm{~d}(\Delta h=26 \mathrm{~mm})$, bifurcated cracks did not emanate from the same horizontal location from where the mode-I crack (in the support layer) first interacts with the interface; instead, there is a shift in the positive $x$ direction from where it reinitiates in the impacted layer. A likely reason is that the location from where the bifurcated cracks emanate is strongly influenced by intrinsic material flaws that exists randomly along the bottom face (affixed to the interlayer) of the impacted layer. The critical condition that governs crack branching, or bifurcation, will be discussed later in section 4. It is clear from Figs. 4, 5 and 6 that the presence of an interface does not always lead to crack branching; however, the location of the interface (relative to the bottom face of the laminate glass panel) appears to have a strong influence over whether mixed-mode cracks would initiate in the impacted layer- this finding appears consistent with the experimental observations made by Sundaram and Tippur (2016b). 
An inspection of the third(c) and fifth(e) frames of Fig. 5 reveal a considerable delay time (also known as crack arrest time) of $52.4 \mu \mathrm{s}$ between the arrival of the initial mode-I crack (in the support layer) at the interface and the reappearance of bifurcated cracks in the impacted layer. This was found to be $66.6 \mu \mathrm{s}$ in Fig. 6, where $\Delta h=36 \mathrm{~mm}$. The delay time is a direct consequence of interactions between the crack-tip plastic zone and interface. Sugimura et al. (1995) found that crack growth encounters a sharp rise in resistance as it approaches a medium that develops a smaller plastic zone size compared to one (the interface) which the crack is currently propagating; in our case, the impacted glass layer does not develop any significant plastic zone size in the vicinity of the crack tip. This delay time is better explained by examining the temporal evolution of the dynamic stress intensity factor - to be presented later in Fig. 8 - which has to increase at a finite rate until it reaches a threshold needed to initiate crack branching (Theocaris and Milios, 1981; Dally and Kobayashi, 1978) in the impacted layer.

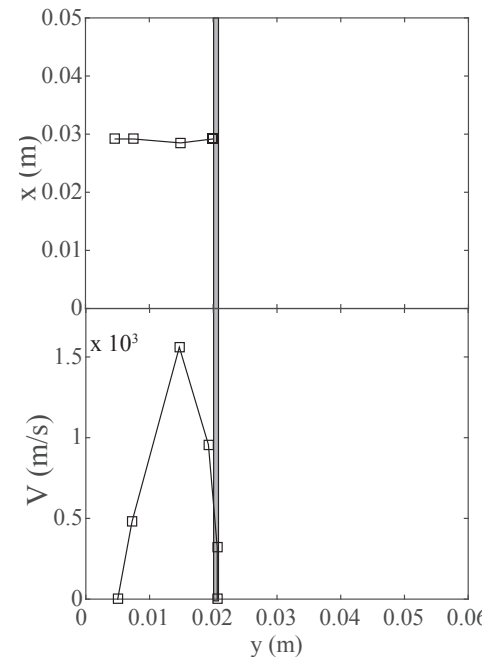

(a) $\Delta h=16 \mathrm{~mm}$

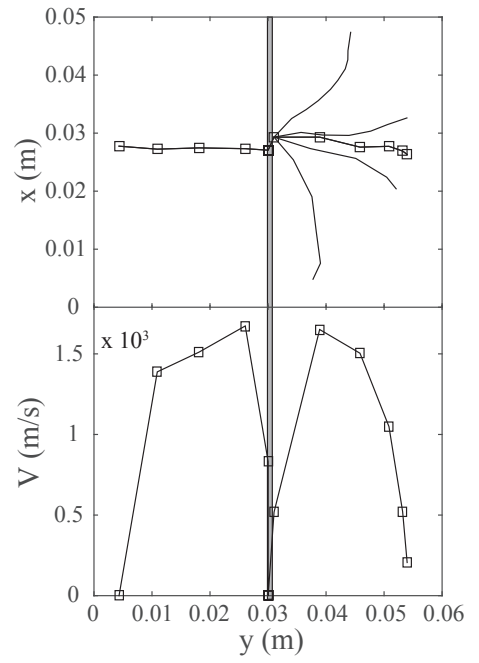

(b) $\Delta h=26 \mathrm{~mm}$

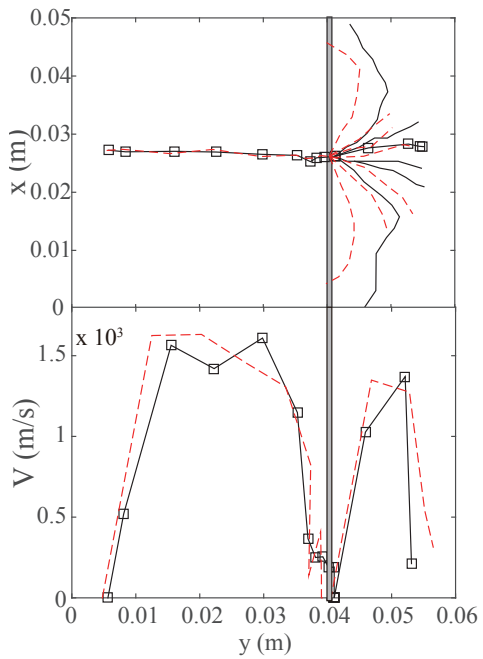

(c) $\Delta h=36 \mathrm{~mm}$

Figure 7: Evolution of crack profile and crack velocity for specimens where: (a) $\Delta h=16$ $\mathrm{mm}$; (b) $\Delta h=26 \mathrm{~mm}$ and (c) $\Delta h=36 \mathrm{~mm}$. The grey column denotes the location of the interface. The origin of the $x$ - and $y$-axes corresponds to the bottom right-hand corner of the caustic images. - - shows repeat test results for $\Delta h=36 \mathrm{~mm}$.

Figure 7 plots the crack trajectory and crack-tip velocity for the three specimens shown in evaluated by dividing the distance between the positions of the crack tip in two consecutive images by the interframe time interval as follows: $V=\sqrt{\left(x_{n}-x_{n-1}\right)^{2}+\left(y_{n}-y_{n-1}\right)^{2}} / \Delta t$. The mode-I crack velocity in the support layer follows a broadly similar pattern regardless of $\Delta h$ : the crack velocity increases abruptly, followed by an equally steep drop as the crack tip approaches the interface. After a time delay, due to interactions between the mode- 
I crack and the interface, bifurcated mixed-mode cracks reappear in the impacted layer, where its velocity (of a mixed-mode crack in the middle) increases rapidly to a (lower) peak value (compared to the preceding peak value) before decreasing monotonically as the crack propagates away from the interface. The measured maximum crack velocities were found to be reasonable since they are lower than the Rayleigh wave velocity in glass of $c_{R}=3370 \mathrm{~m} / \mathrm{s}$ (Sharon et al., 2002). Repeat test was performed for the specimen with $\Delta h=36 \mathrm{~mm}$ and the results plotted in Fig. 7c. It confirms a high degree of reproducibility in the fracture behaviour and in our measurements, both in terms of the maximum crack velocity in the impacted and support layers, as well as the crack arrest time.

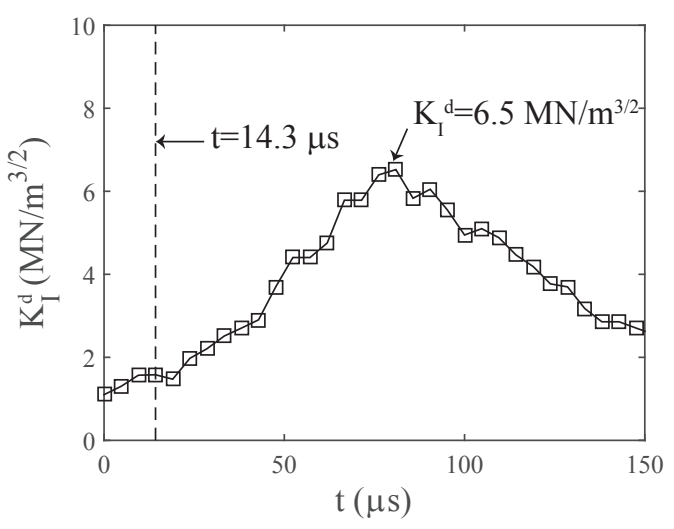

(a) $\Delta h=16 \mathrm{~mm}$

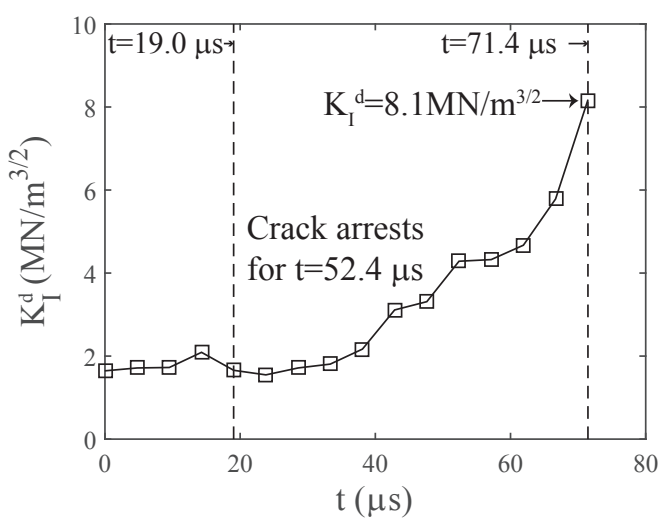

(b) $\Delta h=26 \mathrm{~mm}$

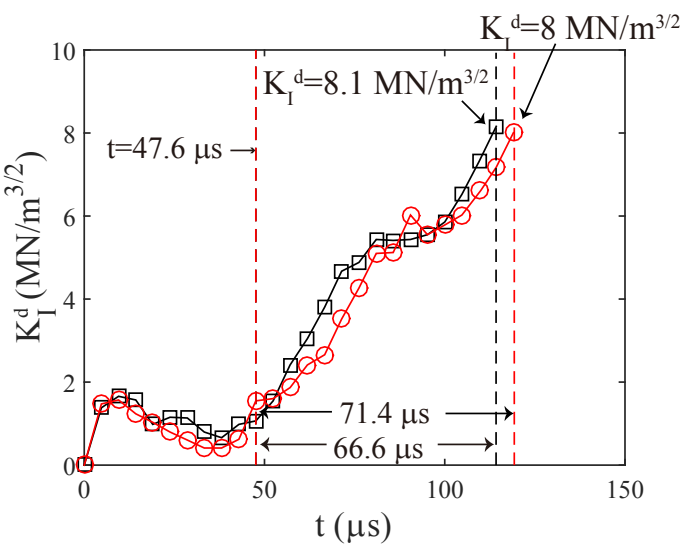

(c) $\Delta h=36 \mathrm{~mm}$

Figure 8: Temporal evolution of dynamic stress intensity factor $K_{I}^{d}$ for specimens with interface located at: (a) $\Delta h=16 \mathrm{~mm}$, (b) $\Delta h=26 \mathrm{~mm}$ and (c) $\Delta h=36 \mathrm{~mm}$. - - (left to right) respectively mark the time instant when crack arrives at and re-emerges from the interface. -0 - shows repeat test results for $\Delta h=36 \mathrm{~mm}$.

The temporal evolution of the dynamic stress intensity factors $K_{I}^{d}(t)$, evaluated based on Eq. 
1, are plotted in Figs. 8a, 8b and 8c for the different interface locations. In all cases, their $K_{I}^{d}(t)$ histories are largely similar before the initial mode-I crack arrives at the interface : it increases initially followed by a drop as the crack approaches the interface. Beyond that their $K_{I}^{d}(t)$ history are very different. In the case of $\Delta h=16 \mathrm{~mm}, K_{I}^{d}(t)$ increases before reducing again. By contrast, for $\Delta h=26$ and $\Delta h=36 \mathrm{~mm}$, there is a monotonic increase of $K_{I}^{d}(t)$ with time - this is also evident by comparing the diameter of caustic spots between Fig. $5 \mathrm{c}$ and $5 \mathrm{~d}$ (also between Fig. $6 \mathrm{c}$ and $6 \mathrm{~d}$ ) - until a critical value of $8.1 \mathrm{MN} / \mathrm{m}^{3 / 2}$ whereupon mixed-mode cracks emanate from the impact layer. The results (red line) from a repeat test measurement of the dynamic stress intensity factor are plotted in Fig. 8c. Their entire $K_{I}^{d}(t)$ time-histories are very close which, again, indicates that the results are reproducible, despite the highly transient nature of the problem and intrinsic flaws that are present in glass specimens.

\subsection{Effects of impact kinetic energy $E_{0}$}

Figures 9, 10 and 11 plot the temporal location of caustic spots, crack velocity and stress intensity factor histories for two different impact kinetic energies of 0.37 and $0.74 \mathrm{~J}$, with corresponding impact velocity of 0.99 and $1.4 \mathrm{~m} / \mathrm{s}$, respectively. Note that both test specimens have identical total height $h_{t}=40.79 \mathrm{~mm}$ and interface location $\Delta h=16 \mathrm{~mm}$. Here, a higher recording frequency of 300,000 frames per second, compared to the first set of experiments, was used to compensate for the smaller total specimen height. Crack branching appeared in the impacted layer of the specimens at both impact kinetic energies. Similar to the findings shown in Figs. 5, 7b and 8b (also Figs. 6, 7c and 8c), where crack branching also occurred, the following observations can be made: (1) The multiple mixed-mode cracks that develop in the impacted layer do not always maintain global symmetry relative to the original mode-I crack plane in the support layer. Again, this is because the location where the crack emanates is highly sensitive to intrinsic flaws that are present in the glass plies; (2) There is a similarly sharp increase and decrease of the mode-I crack-tip velocity as it approaches the interface from the support layer; (3) A similar pattern of dramatic rise-and-fall of the main mixed-mode crack velocity is also recorded as it propagates away from the interface in the impacted layer. Similarly, a higher crack velocity is recorded in the support layer compared to the impacted one; and (4) Branching does not occur until $K_{I}^{d}$ exceeds a critical value of $K_{I}^{d}=7.9$ and $7.6 \mathrm{MN} / \mathrm{m}^{3 / 2}$ which is evident from Figs. 11a and $11 \mathrm{~b}$, respectively.

It is interesting to note that the time it took for the mode-I crack to reach the interface $(t=13.3 \mu \mathrm{s})$ is identical for both impact kinetic energies - see Figs. 11a and 11b - and this is relatively close to $t=14.3 \mu \mathrm{s}$ recorded in Fig. 8a. Note that an identical crack arrival time (at the interface) is almost impossible to achieve for the tests presented here and those in section 3.1, by virtue of the time interval difference (of 4.76 and $3.33 \mu$ s respectively). The time for the mode-I crack to reach the interface is insensitive to the total height of the specimen or to the impact kinetic energy. Unsurprisingly, it depends on the interface location relative to the bottom face of the glass specimen (compare Figs. 4, 5 and 6). 


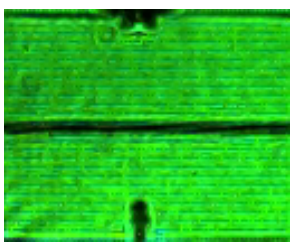

$\mathrm{t}=0 \mu \mathrm{s}$

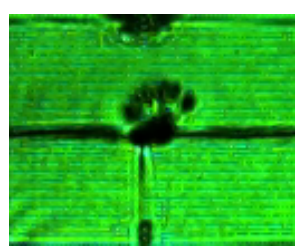

$\mathrm{t}=263.3 \mu \mathrm{s}$

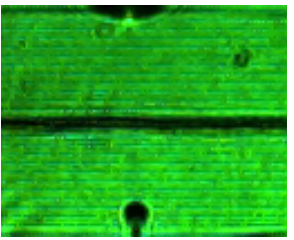

$\mathrm{t}=0 \mu \mathrm{s}$

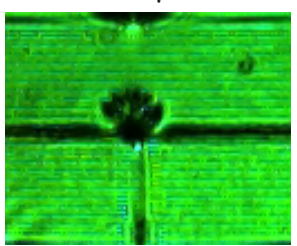

$\mathrm{t}=176.7 \mu \mathrm{s}$

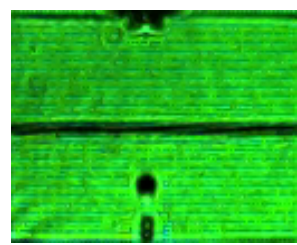

$\mathrm{t}=3.3 \mu \mathrm{s}$

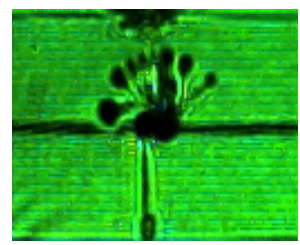

$\mathrm{t}=266.7 \mu \mathrm{s}$

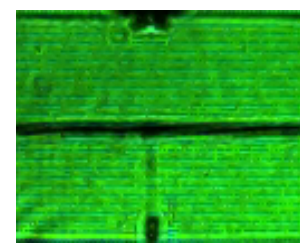

$\mathrm{t}=13.3 \mu \mathrm{s}$

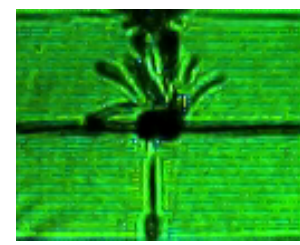

$\mathrm{t}=270.0 \mu \mathrm{s}$

(a) $E_{0}=0.37 \mathrm{~J}$

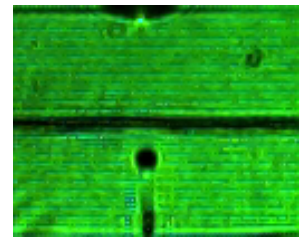

$\mathrm{t}=6.7 \mu \mathrm{s}$

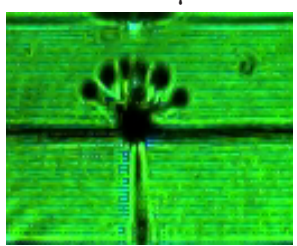

$\mathrm{t}=180.0 \mu \mathrm{s}$

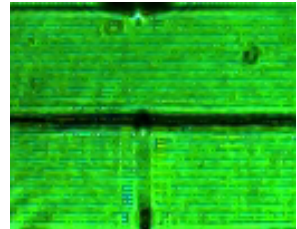

$\mathrm{t}=13.3 \mu \mathrm{s}$

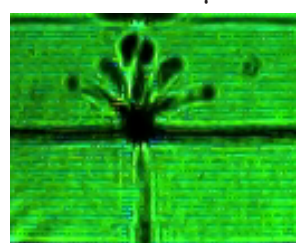

$\mathrm{t}=183.3 \mu \mathrm{s}$

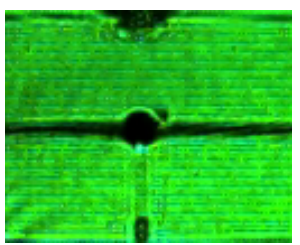

$\mathrm{t}=260.0 \mu \mathrm{s}$

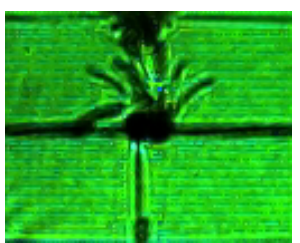

$\mathrm{t}=273.3 \mu \mathrm{s}$

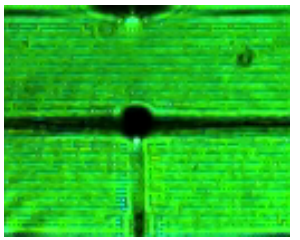

$\mathrm{t}=166.5 \mu \mathrm{s}$

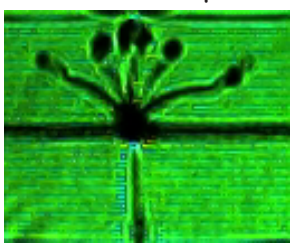

$\mathrm{t}=213.3 \mu \mathrm{s}$

(b) $E_{0}=0.74 \mathrm{~J}$

Figure 9: Temporal evolution of caustic spots in specimens $\left(\Delta h=16 \mathrm{~mm}, h_{t}=40.79 \mathrm{~mm}\right)$ subjected to different impact kinetic energies of (a) $E_{0}=0.37 \mathrm{~J}$ and (b) $E_{0}=0.74 \mathrm{~J}$.

226

Increasing impact kinetic energy leads to a reduction in the delay time for mixed-mode cracks to initiate in the impacted layer, as seen in Fig. 11. The consequence is that there is less time for the dynamic stress intensity factor to reach its critical value as impact kinetic energy increases.

\section{Discussions}

Table 2 summaries the different damage mode that had developed in the SGP laminated glass for various interface locations $\Delta h$, total specimen height $h_{t}$ and impact kinetic energy $E_{0}$. It is clear that cracking in the impacted layer occurs only by branching rather than continuous 


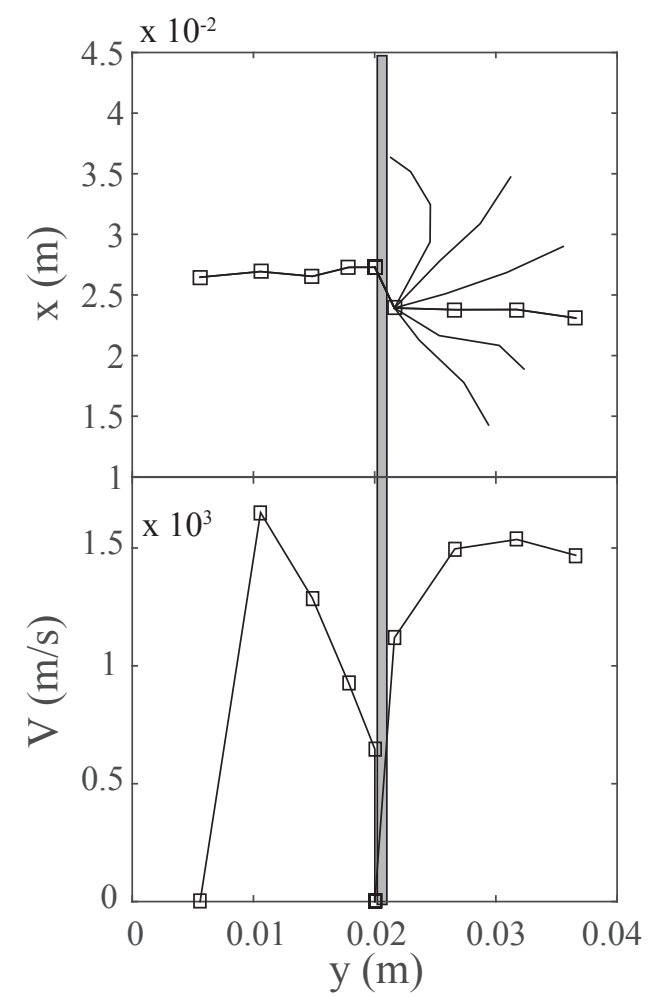

(a) $E_{0}=0.37 \mathrm{~J}$

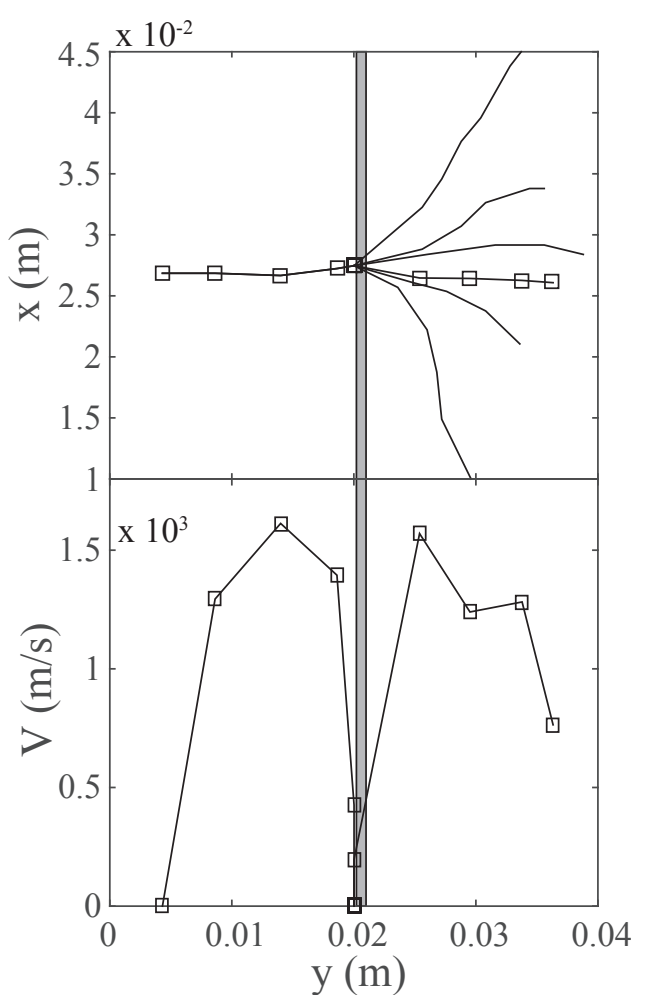

(b) $E_{0}=0.74 \mathrm{~J}$

Figure 10: Evolutions of crack profile and crack velocity: (a) $E_{0}=0.37 \mathrm{~J}$ and (b) $E_{0}=0.74 \mathrm{~J}$. The origin of the $x$ - and $y$-axes corresponds to the bottom right-hand corner of the caustic images.

penetration, at least for the systems studied here where the interface thickness was $0.79 \mathrm{~mm}$. This is consistent with experimental results reported by Park and Chen (2011) where it was found that penetration occurs only when the interface has a near zero thickness. It is worth highlighting that apart from penetration and branching, delamination (or interfacial crack growth) is another possible damage mode, see for example Sundaram and Tippur (2016b,a); Alam et al. (2017) to name a few, that were not observed in our tests. In our experiments, strong adhesives were applied between the interface and glass layers in order to minimise delamination; post-mortem inspection of recovered test specimens confirmed that none had deformed by delamination as expected.

The damage mode listed in Table 2, may be explained from an energy dissipation perspective. Energy accumulation at the crack tip and energy release rate are two important criteria that controls crack branching (Freund, 1990). For mode I fracture, the energy release rate can be written explicitly as a function of the dynamic stress intensity factor $\left(K_{I}^{d}\right)$ and crack speed 


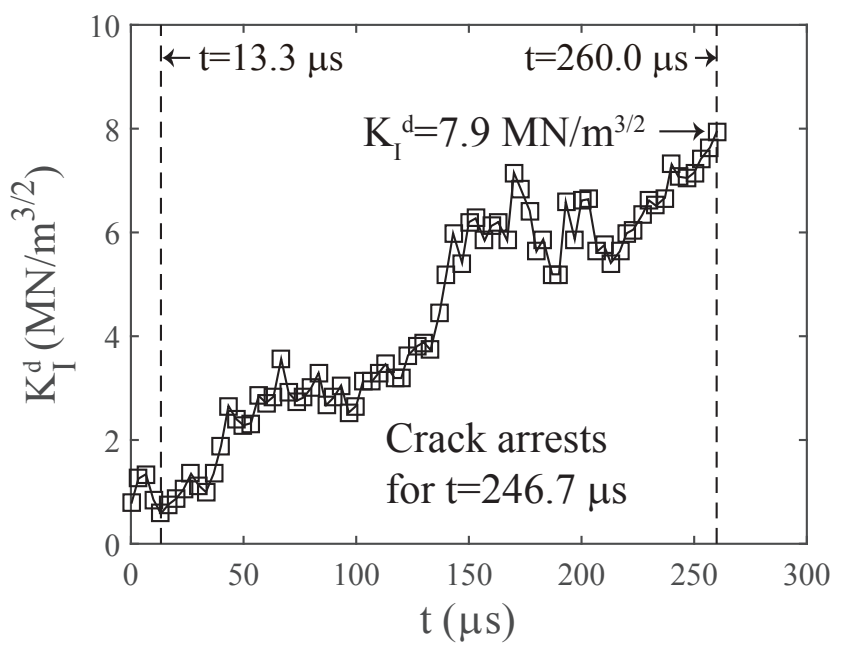

(a) $E_{0}=0.37 \mathrm{~J}$

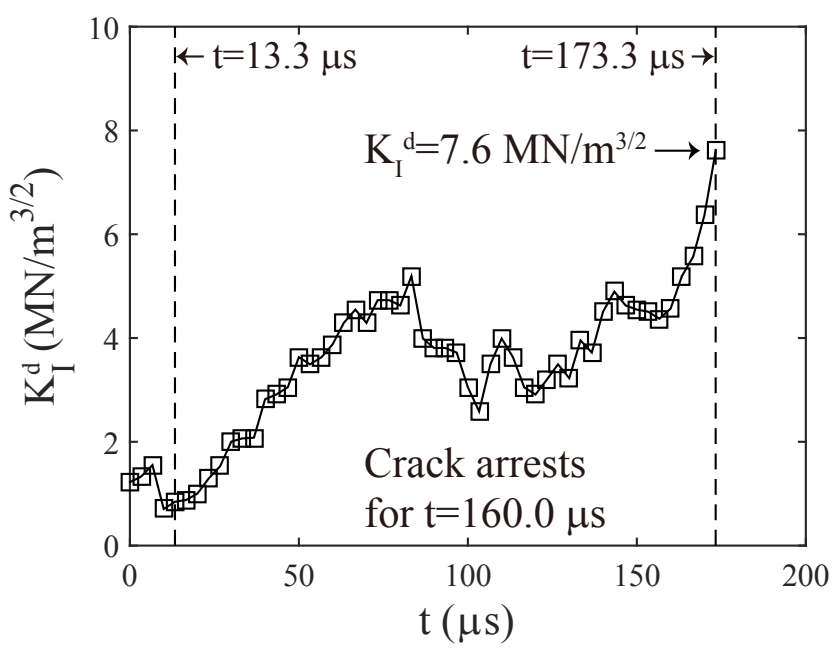

(b) $E_{0}=0.74 \mathrm{~J}$

Figure 11: Temporal evolution of dynamic stress intensity factor $K_{I}^{d}$ at different impact kinetic energy of: (a) $E_{0}=0.37 \mathrm{~J}$ and (b) $E_{0}=0.74 \mathrm{~J}$. - - (left to right) respectively mark the time instant when crack arrives at and re-emerges from the interface.

$247(V)$ as follows (Park and Chen, 2011; Parab and Chen, 2014):

$$
\gamma=\frac{d \Pi}{d l}=\frac{1+\nu}{E} \frac{V^{2} \alpha_{d}}{C_{s}^{2} R(V)}\left(K_{I}^{d}\right)^{2}
$$

${ }_{248}$ where $\Pi, l, C_{s}, C_{d}$ are energy dissipation, crack length, shear wave speed and dilatational 
Table 2: Mode of damage by the SGP laminated glass.

\begin{tabular}{cccccc}
\hline $\begin{array}{c}\text { Specimen } \\
\text { number }\end{array}$ & $\begin{array}{c}\text { Interface location } \\
\Delta h(\mathrm{~mm})\end{array}$ & $\begin{array}{c}\text { Total specimen } \\
\text { height, } h_{t}(\mathrm{~mm})\end{array}$ & $\begin{array}{c}\text { Impact kinetic } \\
\text { energy, } E_{0}(\mathrm{~J})\end{array}$ & $\begin{array}{c}\text { MDSIF } \\
\left(\mathrm{MN} / \mathrm{m}^{3 / 2}\right)\end{array}$ & $\begin{array}{c}\text { Damage } \\
\text { mode }\end{array}$ \\
\hline 1 & 16 & 60.79 & 1.47 & 6.5 & Inhibition \\
2 & 26 & 60.79 & 1.47 & 8.1 & Branching \\
3 & 36 & 60.79 & 1.47 & 8.1 & Branching \\
$3^{*}$ & 36 & 60.79 & 1.47 & 8.0 & Branching \\
4 & 16 & 40.79 & 0.37 & 7.9 & Branching \\
5 & 16 & 40.79 & 0.74 & 7.6 & Branching \\
\hline
\end{tabular}

MDSIF: maximum dynamic stress intensity factor.

speed respectively; $R(V)=4 \alpha_{d} \alpha_{s}-\left(1+\alpha_{s}^{2}\right)^{2}$ is the Rayleigh function, $\alpha_{d}=\sqrt{1-V^{2} / C_{d}^{2}}$ and $\alpha_{s}=\sqrt{1-V^{2} / C_{s}^{2}}$. The initial mode-I crack velocity becomes negligibly small when it reaches the interface. Just before mixed-mode cracks emanate from the impacted layer, the dynamic stress intensity factor $\left(K_{I}^{d}\right)$ is the dominant term in Eq.2. Therefore, as $K_{I}^{d}$ increases, $\gamma$ also increases with time until the critical value of $K_{I}^{d}$ is reached for mixed-mode cracks to initiate in the impacted layer. In this case, a simple maximum dynamic stress intensity factor criterion may be employed to determine whether crack branching would develop. If the development of a single crack in the impacted layer is not sufficient to dissipate the accumulated energy through creation of new crack surfaces, then instability such as crack branching would occur in the impacted layer; this explains the preference for branching damage mode recorded in our experiments.

The caustic method is used here, for the first time, to measure the dynamic stress intensity factor at the crack tip in SGP laminated glass; it provides confirmation on the applicability of a simple criterion, based on the maximum dynamic stress intensity factor (or MDSIF for brevity), to predict crack branching. It should be noted that only the dynamic SIF of the mode-I crack in the support layer was measured. However, the dynamic SIF of the mixedmode branched cracks cannot be extracted because the corresponding caustic spots were not consistently large enough for diameter measurement due to intrinsic problems associated with glass (Takahashi, 1999). Notwithstanding, one should expect the dynamic stress intensity factors of the mixed-mode cracks to be significantly lower than the critical dynamic stress intensity factor for crack branching: this is evident through a cursory comparison of the diameter of the caustic spots before and after branching in Figs. 5, 6 and 9.

Table 2 also lists the MDSIF that were extracted from Figs. 8 and 11. For the case of $\Delta h=16, h_{t}=60.79$ and $h_{d}=200 \mathrm{~mm}$, its MDSIF is considerably lower compared to the rest and did not reach the critical value needed for branching to initiate in the impacted layer, which explains why the mode-I crack was arrested at the interface. The current 
experimental results suggest a possible strategy for damage containment (within the support layer) by altering the interface location to prevent branched cracks from emanating in the impacted layer. The fact that the MDSIF is nominally similar, between the cases where crack branching were observed, indicates that the critical value of $K_{I}^{d}$ is largely unaffected by the interface location, total specimen height or impact kinetic energy.

Although a simple MDSIF criterion appears successful in predicting the mode of deformation, viz. either crack inhibition or branching, a mechanistic explanation as to how a smaller interface and pre-crack tip distance $(\Delta h)$ facilitate crack containment is still lacking. It is worth emphasizing that in the present study, $t=0 \mu \mathrm{s}$ corresponds to the instant when cracking initiates at the pre-crack tip. This initial phase of the impact event was excluded from our analysis when studying the crack-interface interaction. It is conjectured that the total energy in the system (Specimens 1-3) at $t=0 \mu \mathrm{s}$ (as we had currently defined) might not, in fact, be identical even though they have identical $E_{0}$. The partitioning of $E_{0}$ between the various constituents that made up the laminated glass panel (due to the different interface location) during this initial phase is likely to play a significant role in the subsequent crack propagation behaviour. Numerical simulations are needed to rationalise the results reported here by examining the energy exchange history to include the pre- and post- crack initiation phases; this is part of a current investigation to be reported elsewhere. The results reported here will be useful for the development and validation of the finite-element model needed to perform the aforesaid parametric investigations. The topic of how to accurately measure the mixed-mode stress intensity of glass, using the optical caustic method, is also part of an on-going investigation.

\section{Conclusions}

The effects of interface location and impact kinetic energy upon the damage regime that develop in SGP laminated glass were investigated in this paper through optical measurements using a caustic image method. Dynamic stress intensity factor along with crack morphology and crack velocity histories were measured. The results showed that the initial mode-I crack is permanently arrested by the interface if its distance from the initial pre-crack tip is below a critical distance. Above this critical distance, the mode-I crack was only arrested momentarily before bifurcated mixed-mode cracks appear in the impacted layer. The crack arrest time at the interface is a consequence of a sharp rise in resistance to propagation by a crack as it approaches a medium that develops a smaller plastic zone size compared to one which it is currently propagating. The arrest time reduces significantly with increasing impact kinetic energy. The crack at the interface, prior to branching, allows the stress intensity factor to increase, leading to energy accumulation at the crack tip. Dissipation of this accumulated energy causes crack branching, which was shown to be predicted by a maximum dynamic stress intensity factor criterion. The critical value of dynamic stress intensity factor that must be reached for crack branching to develop in the impacted layer was found to be insensitive interface location, specimen total height and initial kinetic energy. 


\section{Acknowledgment}

The authors are indebted to Prof. Qing Zhou at Tsinghua University for the invaluable discussions. This work is financially supported, in part, by National Science Foundation of China [Grant No. 11372164 and No. 11772176] and, in part, by Tsinghua University [Grant No. 20132001016]. PJ Tan acknowledges the financial support by QinetiQ, UK (Mr Robert Ball - Structures \& Survivability, Platform Design and Life Support IDT).

\section{References}

Alam, M., Parmigiani, J. P., Kruzic, J. J., 2017. An experimental assessment of methods to predict crack deflection at an interface. Eng. Fract. Mech. 181, 116-129.

Bennison, S., Sloan, J., Kistunas, D., Buehler, P., Amos, T., Smith, C., 2005. Laminated glass for blast mitigation: role of interlayer properties. In: Glass processing days. pp. 17-20.

Chen, J., Xu, J., Yao, X., Liu, B., Xu, X., Zhang, Y., Li, Y., 2013. Experimental investigation on the radial and circular crack propagation of PVB laminated glass subject to dynamic out-of-plane loading. Eng. Fract. Mech. 112-113, 26-40.

Chen, J., Xu, J., Yao, X., Xu, X., Liu, B., Li, Y., 2014. Different driving mechanisms of in-plane cracking on two brittle layers of laminated glass. Int. J. Impact Eng. 69, 80-85.

Clegg, W. J., Kendall, K., Alford, N. M., Button, T. W., Birchall, J. D., 1990. A simple way to make tough ceramics. Nature 347 (6292), 455-457.

Dally, J. W., Kobayashi, T., 1978. Crack arrest in duplex specimens. Int. J. Solids Struct. 14 (2), 121 - 129.

Freund, L. B., 1990. Dynamic Fracture Mechanics. New York: Cambridge University Press.

Gan, F., 1964. Optic Glass (in Chinese). Beijing: Beijing Scientific Publishing.

Hao, W., Sheng, X., Guo, G., Chen, X., Zhu, J., 2016a. Mixed mode dynamic crack-fiber bundle interaction using caustics. Polym. Test. 55, 230-237.

Hao, W., Tang, C., Yuan, Y., Ma, Y., 2016b. Investigation of dynamic mode I matrix crack-fiber bundle interaction in composites using caustics. Composites Part B: Engineering 92, 395-404.

Hao, W., Yao, X., Ma, Y., Yuan, Y., 2015. Experimental study on interaction between matrix crack and fiber bundles using optical caustic method. Eng. Fract. Mech. 134, 354-367.

He, M. Y., Hutchinson, J. W., 1989. Crack deflection at an interface between dissimilar elastic materials. Int. J. Solids Struct. 25 (9), $1053-1067$.

Lee, J. J.-W., Lloyd, I. K., Chai, H., Jung, Y.-G., Lawn, B. R., 2007. Arrest, deflection, penetration and reinitiation of cracks in brittle layers across adhesive interlayers. Acta Mater. 55 (17), 5859-5866.

Liu, B., Xu, T., Xu, X., Wang, Y., Sun, Y., Li, Y., 2016. Energy absorption mechanism of polyvinyl butyral laminated windshield subjected to head impact: Experiment and numerical simulations. Int. J. Impact Eng. $90,26-36$.

Needleman, A., 2018. Dynamic mode ii crack growth along an interface between an elastic solid and a plastic solid. J. Mech. Phys. Solids (In press).

Papadopoulos, G. A., 1993. Fracture Mechanics, in: The Experimental Method of Caustics and the Det.Criterion of Fracture. London: Springer.

Parab, N. D., Chen, W. W., 2014. Crack Propagation Through Interfaces in a Borosilicate Glass and a Glass Ceramic. Int. J. Appl. Glass Sci. 5 (4), 353-362.

Park, H., Chen, W. W., 2011. Experimental Investigation on Dynamic Crack Propagating Perpendicularly Through Interface in Glass. J. Appl. Mech 78 (5), 051013-051013-10.

Sharon, E., Cohen, G., Fineberg, J., 2002. Crack front waves and the dynamics of a rapidly moving crack. Phys. Rev. Lett. 88, 085503.

Shaw, M., Marshall, D., Dadkhah, M., Evans, A., 1993. Cracking and damage mechanisms in ceramic/metal multilayers. Int. J. Fract. 41 (11), $3311-3322$. 
Siegmund, T., Fleck, N., Needleman, A., 1997. Dynamic crack growth across an interface. Int. J. Fract. $85(4), 381-402$.

Sugimura, Y., Grondin, L., Suresh, S., 1995. Fatigue crack growth at arbitrary angles to bimaterial interfaces. Scr. Metall. Mater. 33 (12), 2007-2012.

Sundaram, B., Tippur, H., 2016a. Dynamic crack growth normal to an interface in bi-layered materials: An experimental study using digital gradient sensing technique. Exp. Mech. 56 (1), 37-57.

Sundaram, B. M., Tippur, H. V., 2016b. Dynamics of crack penetration vs. branching at a weak interface: An experimental study. J. Mech. Phys. Solids 96, 312-332.

Sundaram, B. M., Tippur, H. V., 2017. Dynamic mixed-mode fracture behaviors of PMMA and polycarbonate. Eng. Fract. Mech. 176, 186-212.

Takahashi, K., 1999. Fast fracture in tempered glass. In: Time Dependent Mechanical Response of Engineering Ceramics. Vol. 166 of Key Engineering Materials. Trans Tech Publications, pp. 9-18.

Tang, C., 2014. Experimental investigation of the fiber bundle shielding effect on the dynamic matrix crack using optical caustic method. Polym. Test. 40, 46-53.

Theocaris, P. S., 1970. Local yielding around a crack tip in plexiglas. J. Appl. Mech. 37 (2), 409-415.

Theocaris, P. S., Demakos, C. B., 1986. Crack bifurcation modes in composite plates under impact. Int. J. Fract. 32 (2), 71-92.

Theocaris, P. S., Milios, J., 1981. Crack-arrest at a bimaterial interface. Int. J. Solids Struct. 17 (2), 217 230.

Xu, L., Rosakis, A. J., 2003. An experimental study of impact-induced failure events in homogeneous layered materials using dynamic photoelasticity and high-speed photography. Opt. Lasers Eng. 40 (4), 263 - 288.

Xu, X., Liu, B., Li, Y., 2016. Investigation on Dynamic Propagation Characteristics of In-Plane Cracks in PVB Laminated Glass Plates. Adv. Mater. Sci. Eng. 2016, 1-13.

$\mathrm{Xu}, \mathrm{X} ., \mathrm{Xu}, \mathrm{J} ., \mathrm{Chen}, \mathrm{J} ., \mathrm{Li}, \mathrm{P} ., \mathrm{Liu}, \mathrm{B} ., \mathrm{Li}, \mathrm{Y} ., 2$ 2017. Investigation of dynamic multi-cracking behavior in PVB laminated glass plates. Int. J. Impact Eng. 100, 62-74.

Yan, Y., Huang, K., Sumigawa, T., Kitamura, T., 2018. Fracture criterion of mixed-mode crack propagation along the interface in nanoscale components. Eng. Fract. Mech. (In press).

Yao, X., Chen, J., Jin, G., Arakawa, K., Takahashi, K., 2004. Caustic analysis of stress singularities in orthotropic composite materials with mode-I crack. Compos. Sci. Technol. 64 (7), 917-924.

Yao, X., Xu, W., Xu, M., Arakawa, K., Mada, T., Takahashi, K., 2003. Experimental study of dynamic fracture behavior of pmma with overlapping offset-parallel cracks. Polym. Test. 22 (6), 663 - 670.

Yao, X. F., Xu, W., 2011. Recent application of caustics on experimental dynamic fracture studies. Fatigue \& Fracture of Engineering Materials \& Structures 34 (6), 448-459.

Yao, X. F., Xu, W., Yeh, H. Y., 2007. Investigation of crack tip evolution in functionally graded materials using optical caustics. Polym. Test. 26 (1), 122-131.

Yuan, Y., Tan, P. J., Li, Y., 2017a. Dynamic structural response of laminated glass panels to blast loading. Compos. Struct. 182, 579-589.

Yuan, Y., Xu, C., Xu, T., Sun, Y., Liu, B., Li, Y., 2017b. An analytical model for deformation and damage of rectangular laminated glass under low-velocity impact. Compos. Struct. 176, 833-843.

Zhang, J. L., Bai, S. L., Liu, D. L., Zhang, Q. X., Yu, Z. Z., 2007. Caustic study on stress singularities in polypropylene/CaCO3 nanocomposites with nonionic modifier. Compos. Sci. Technol. 67 (2), 238-243.

Zhang, X., Hao, H., Ma, G., 2013. Laboratory test and numerical simulation of laminated glass window vulnerability to debris impact. Int. J. Impact Eng. 55, $49-62$.

Zhang, X., Shi, Y., Hao, H., Cui, J., 2015. The mechanical properties of ionoplast interlayer material at high strain rates. Materials \& Design 83, 387-399. 